GEOPHYSICAL RESEARCH LETTERS, VOL. 20, NO. 23, PAGES 2767-2770, DECEMBER 14, 1993

\title{
PLASMA WAVES OBSERVED AT LOW ALTITUDES IN THE TENUOUS VENUS NIGHTSIDE IONOSPHERE
}

\author{
R. J. Strangeway, C. T. Russell, and C. M. $\mathrm{Ho}^{\dagger}$
}

Institute of Geophysics and Planetary Physics, University of California at Los Angeles

\section{H. Brace}

Space Physics Research Laboratory, The University of Michigan

\begin{abstract}
The Pioneer Venus Orbiter Electric Field Detector (OEFD) measured many plasma wave bursts throughout the low altitude ionosphere during the final entry phase of the spacecraft. Apart from $100 \mathrm{~Hz}$ bursts observed at very low altitudes $(\sim 130 \mathrm{~km})$, the bursts fall into two classes. The first of these is a wideband signal that is observed in regions of low magnetic field, but average densities, in comparison to the prevailing ionospheric condition. This wideband signal is not observed in the $30 \mathrm{kHz}$ channel of the OEFD, but is restricted to the $5.4 \mathrm{kHz}$ channel and lower. Since these bursts are observed with roughly constant burst rate above $160 \mathrm{~km}$ altitude, we attribute them to ion acoustic mode waves generated by precipitating solar wind electrons. The second type of signal is restricted to $100 \mathrm{~Hz}$ only, and is observed in regions of low electron beta, consistent with whistler-mode waves. These waves could be generated by lightning in the Venus atmosphere if the vertical component of the magnetic field $>3.6 \mathrm{nT}$. Unfortunately, the spacecraft spin axis is mainly horizontal, and only that component of magnetic field can be measured. Alternatively, the $100 \mathrm{~Hz}$ bursts could be generated locally through gradient drift instabilities, provided the ambient magnetic field is horizontal. Because the ionosphere is very different during the entry phase, compared to the ionosphere as observed early in the Pioneer Venus mission, any conclusions regarding the source of the plasma waves detected during entry phase cannot be applied directly to the earlier observations.
\end{abstract}

\section{Introduction}

The last periapsis data before atmospheric entry of the Pioneer Venus Orbiter were acquired on October 7, 1992. Immediately prior to this, there were some 50 passes of the nightside ionosphere of Venus with periapsis altitude $<200$ $\mathrm{km}$. At this time solar activity was waning, while solar activity was high early in the mission $(1979-1980)$, when the spacecraft initially sampled the low altitude nightside ionosphere. The entry phase period therefore provided an opportunity to compare observations at solar intermediate levels with solar maximum. The density of the nightside ionosphere was lower during the entry phase [Theis and Brace, 1993], and the ionosphere was a moderate beta plasma, as

†Now at Jet Propulsion Laboratory.

Copyright 1993 by the American Geophysical Union.

Paper number 93GL02629

0094-8534/93/93GL-02629\$03.00 opposed to high beta at solar maximum [Russell et al., 1993] (beta is the ratio of thermal to magnetic pressure).

As expected, the nightside ionosphere of Venus was quite different during entry phase from that observed early in the Pioneer Venus mission. In this letter we will describe some of the plasma waves observed during the entry phase. Many of the observations are reminiscent of the plasma waves previously reported as being due to atmospheric lightning (see the reviews of Russell [1991] and Strangeway [1991] for a more complete discussion of the early mission plasma wave observations). However, the ionosphere during entry phase is sufficiently dissimilar from the ionosphere at solar maximum that conclusions based on the entry phase observations cannot be applied directly to the earlier observations. In particular, while higher frequency bursts detected during entry phase are almost certainly locally generated, similar (but not identical) bursts observed early in the mission are more likely due to atmospheric lightning.

$100 \mathrm{~Hz}$ bursts are also observed during entry phase. Very intense bursts are detected at lowest altitudes $(\sim 130 \mathrm{~km})$, and these could be the direct measurement of radiation from atmospheric discharges [Strangeway et al., 1993]. $100 \mathrm{~Hz}$ bursts are also detected at higher altitudes. While a lightning source might explain these bursts, they are observed in regions of low electron density, and could be generated by the vertical density gradient in the topside ionosphere.

\section{Data Examples}

Plasma wave data from orbit 5047 (September 29, 1992) are shown in Figure 1. The top four panels give the plasma wave intensity as measured by the four OEFD channels, in descending order, $30 \mathrm{kHz}, 5.4 \mathrm{kHz}, 730 \mathrm{~Hz}$ and $100 \mathrm{~Hz}$. Wave bursts, identified using the technique described by $\mathrm{Ho}$ et al. [1991,1992], are indicated by plus symbols below each wave intensity trace. The spin-axis component of the magnetic field is given below this. In this panel we have drawn a baseline of $-26 \mathrm{nT}$, which is the median magnetic field offset [Russell et al., 1993]. The high resolution electron density, as measured by the Langmuir probe, is shown in the bottom panel as open circles. However, these data have a different temporal resolution than the plasma wave and magnetic field data. In determining the dependence of the wave bursts on the electron measurements we have quadratically interpolated the electron data. The resultant interpolated data are given by the solid line passing through the circles.

Wideband bursts are often detected during the entry phase interval, and have many of the characteristics of the data shown in Figure 1. Another example is given in Figure 
Obit 5047, Sept 29, 1992 - Periapsis tlme 2208:52.05 UT

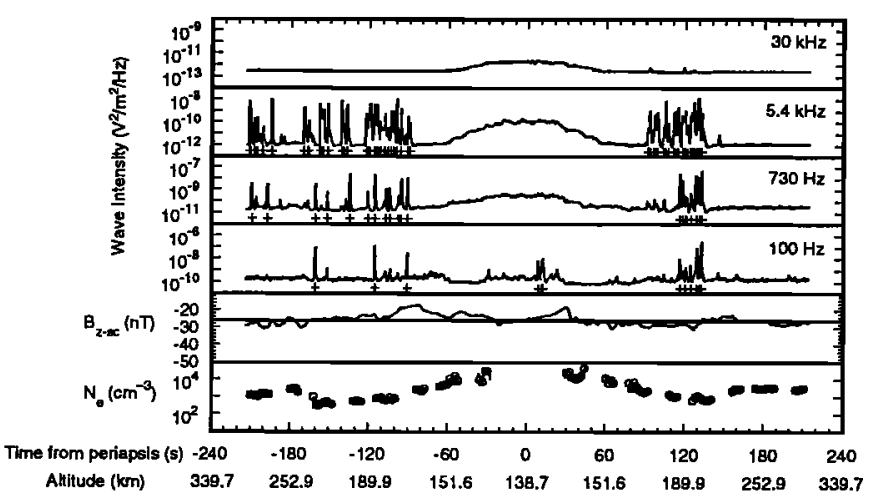

Fig. 1. Plasma wave data acquired on orbit 5047. Eight minutes of data are shown, centered on periapsis. The top four panels show the plasma wave intensity, together with a burst identifier, indicated by the plus symbols. The spin-axis component of the magnetic field is shown below the plasma wave data. The electron data are given in the bottom panel.

2 for orbit 5048 (September 30, 1992). The wideband bursts are observed both inbound and outbound, close to but not at periapsis. Additionally, no bursts occur at $30 \mathrm{kHz}$, unlike the wideband bursts observed near periapsis during the early Pioneer Venus mission [Ho et al., 1991].

Figure 3 shows an example of $100 \mathrm{~Hz}$ bursts observed on orbit 5051 (October 3, 1992) which are in some respects similar to the bursts shown in Figures 1 and 2, in that the bursts are observed both inbound and outbound, but not at periapsis. In this case, however, no bursts occur at higher frequencies. In addition, the spin-axis magnetic field is stronger when the isolated $100 \mathrm{~Hz}$ bursts occur.

One common feature in Figures $1-3$ is the presence of a density trough at altitudes around $200 \mathrm{~km}$, also discussed by Theis and Brace [1993]. There is some indication that the higher frequency waves occur within this trough.

\section{Altitude Profiles}

We have investigated the wave burst dependence on th. ambient plasma parameters as a function of spacecraft altitude, as shown in Figure 4, where the data are binned in 10 $\mathrm{km}$ altitude bins. In panel a) the $100 \mathrm{~Hz}$ burst rate and 5.4 $\mathrm{kHz}$ burst rates are shown. The wideband signals are well

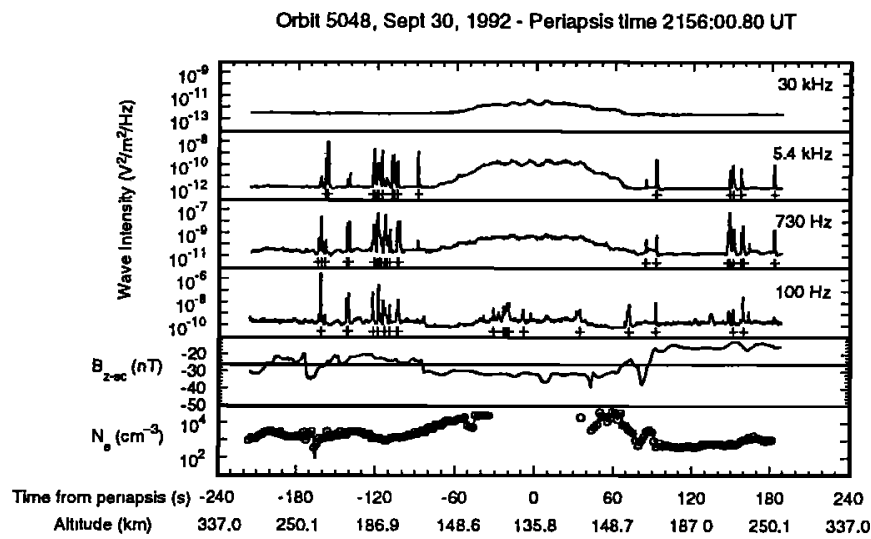

Fig. 2. Plasma wave data acquired on orbit 5048. Similar to Figure 1.
Obil 5051, Oct 3, 1992 - Perlapsis time 2109:36.63 UT

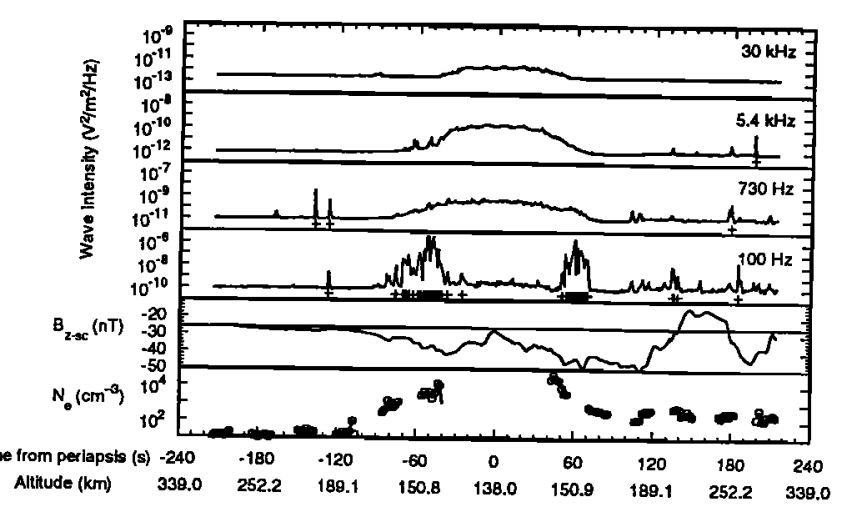

Fig. 3. Plasma wave data acquired on orbit 5051. Similar to Figure 1.

characterized by the $5.4 \mathrm{kHz}$ channel. In calculating the burst rate we have taken into account the different data resolutions used during entry phase. We use an average burst rate at each altitude given by $\Sigma n_{j}\left(1+\Delta t_{j} / \Delta t_{1}\right) / 2 \Sigma N_{j} \Delta t_{j}$, where $n_{j}$ is the number of bursts at a particular data resolution, $N_{j}$ is the total number of samples, $\Delta t_{j}$ is the temporal resolution of the data, and $\Delta t_{1}$ is the shortest sampling interval. This rate is the average of two limiting cases, one that assumes all bursts are isolated, the other that assumes bursts are continuous within a sample. In the latter case we also assume that only one burst occurs per sample at the highest resolution, which was $0.5 \mathrm{~s}$ during entry phase, i.e. $\Delta t_{1}=0.5 \mathrm{~s}$.

In Figure 4 the $100 \mathrm{~Hz}$ burst rate is largest at the lowest altitude. These bursts occurred on the last two orbits of the spacecraft (5054 and 5055) and may be due to atmospheric discharges being detected below the ionospheric density peak [Strangeway et al., 1993]. There is a minimum in the $100 \mathrm{~Hz}$ burst rate, and a second maximum in the $150-160$ $\mathrm{km}$ altitude range. These bursts correspond to signals such as those shown in Figure 3. The $5.4 \mathrm{kHz}$ rate increases to a roughly constant rate above $160 \mathrm{~km}$ altitude. These bursts correspond to wideband bursts as shown in Figures 1 and 2 .

The rectified spin-axis component of the magnetic field is also shown in Figure 4. Prior to rectifying the field we have removed the $-26 n$ T offset. The thick solid line gives the median value of the field for all data samples, while the thin line gives the median value when $100 \mathrm{~Hz}$ bursts occur, and the dotted line gives the median when $5.4 \mathrm{kHz}$ bursts occur. Except at the lowest altitudes, the median field is the same for all the data and for $100 \mathrm{~Hz}$ bursts. On the other hand, the median field is lower when $5.4 \mathrm{kHz}$ bursts occur.

The electron density is shown in panel c) of Figure 4. As noted previously, we have interpolated the electron data so that they have the same temporal resolution as the plasma wave data. We have compared medians of all the interpolated data, binned in $10 \mathrm{~km}$ altitude bins, with the original high resolution data and find the average ratio of the median interpolated data over the median original data $=0.996 \pm$ 0.067 , indicating that the interpolated data are representative of the original data. The electron data do not extend to the lowest altitudes because of possible contamination by ionization due to neutral impacts on the spacecraft.

The electron beta $\left(\beta_{e}\right)$ is plotted in panel d) of Figure 4. In calculating $\beta_{e}$ we have used the electron temperature from 


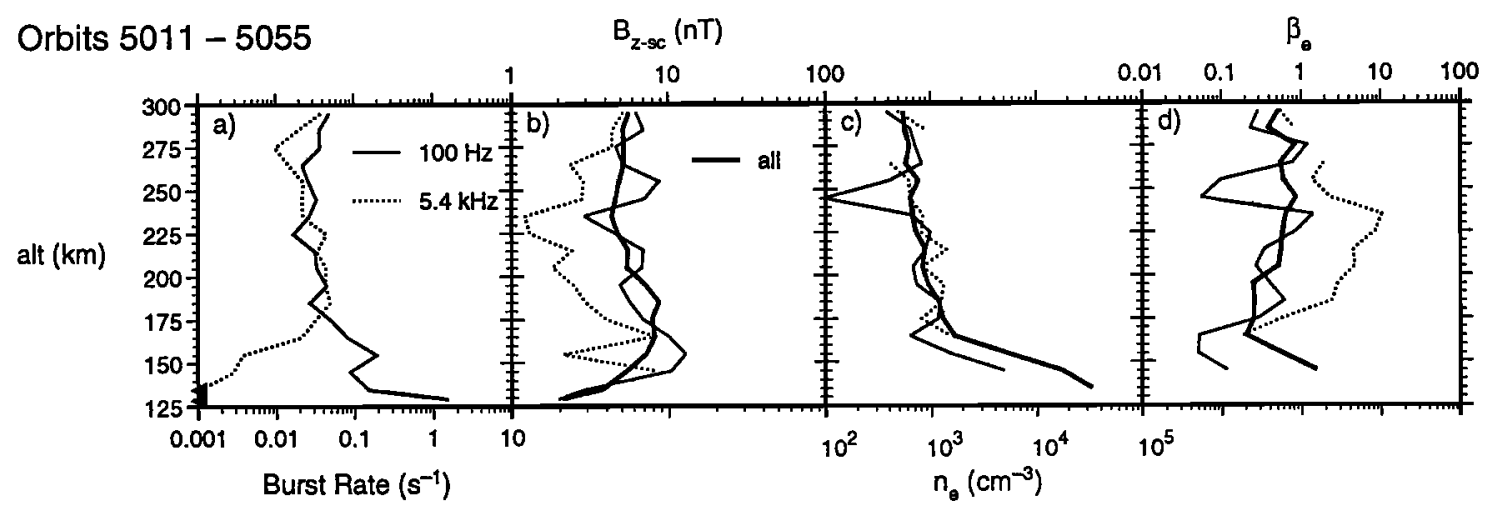

Fig. 4. Altitude profiles of the burst rate and plasma parameters for the entry phase interval (orbits $5011-5055$ ). The burst rate for the $100 \mathrm{~Hz}$ (solid line) and $5.4 \mathrm{kHz}$ (dotted line) channels are shown in panel a). The arrows mark those altitude bins for which the $5.4 \mathrm{kHz}$ burst rate is $<0.001 \mathrm{bursts} / \mathrm{sec}$. The medians of the rectified spin-axis magnetic field (b), electron density (c), and electron beta (d) are also shown. The medians are calculated for all samples (thicker solid line), samples when $100 \mathrm{~Hz}$ bursts occur (thin solid line), and when $5.4 \mathrm{kHz}$ bursts occur (dotted line).

lower (12 s) resolution data. Because these data, referred to as UADS data, are more sparse than the high resolution data we have not interpolated these data. Instead, we have binned the UADS data as a function of altitude and used the same electron temperature profile for all of the $\beta_{e}$ calculations. In addition, following the analysis of Russell et al. [1993], we have multiplied the rectified spin-axis component of the magnetic field by 2.5 to obtain the total magnetic field.

Figure 4 shows that the $100 \mathrm{~Hz}$ bursts occur in regions of $\beta_{e}<1$, indicating that these waves are whistler-mode waves, which are weakly damped in a low $\beta_{e}$ plasma [Strangeway, 1992]. At lowest altitudes, where $\beta_{e}$ is usually larger, the 100 $\mathrm{Hz}$ bursts occur in regions of reduced density and enhanced field. By way of contrast, the $5.4 \mathrm{kHz}$ bursts occur in high beta regions, but this is because the spin-axis component of the ambient field is reduced, rather than an enhancement in density. Again, we note that the magnetometer only measures the spin-axis component of the field, and we assume that the total field is proportional to this component.

Plasma waves were detected at low altitudes during the early part of the Pioneer Venus mission, and we include data from Season III (orbits 484 - 560) in Figure 5, using a similar analysis to that used in Figure 4. The main difference in the analysis is that high resolution temperature data are also available, and we have used these data rather than lower resolution UADS data. Considering the 100 bursts first, we see that the $100 \mathrm{~Hz}$ burst rate decreases slowly with increas- ing altitude. The $100 \mathrm{~Hz}$ bursts occur in regions of enhanced magnetic field and slightly reduced density. As a consequence, the $100 \mathrm{~Hz}$ bursts occur mainly in regions of low $\beta_{e}$, which is required both for whistler-mode propagation from lightning [Strangeway, 1992], and also for generation by the lower hybrid drift instability [Huba, 1992]. In the latter case, however, collisions tend to reduce the instability, suggesting that the lower hybrid drift would be more likely to occur at higher altitudes. The $5.4 \mathrm{kHz}$ bursts, on the other hand, occur mainly at lowest altitude, in regions with somewhat reduced density, but typical magnetic field strengths.

\section{Conclusions}

The nightside ionosphere of Venus is markedly different during the entry phase of the Pioneer Venus mission, in comparison to data acquired earlier in the mission. The plasma beta is of order unity during the entry phase, while the beta was much higher in Season III [Russell et al., 1993].

Duting the entry phase $100 \mathrm{~Hz}$ bursts are found to occur in regions of low electron beta, suggesting that these bursts are whistler-mode. A similar property of the $100 \mathrm{~Hz}$ bursts was also found earlier in the mission. It is therefore possible that $100 \mathrm{~Hz}$ bursts like those shown in Figure 3 could be generated by lightning, although the symmetry of the bursts with respect to periapsis is puzzling. Perhaps the spacecraft encounters a layer of enhanced magnetic field at higher alti-

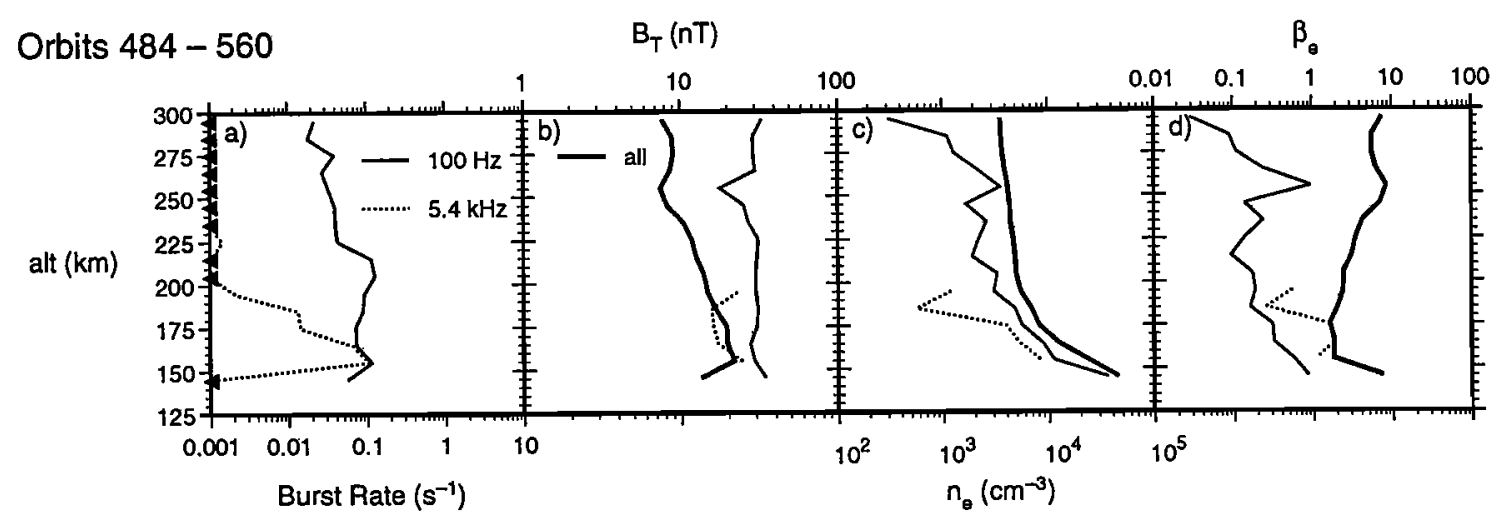

Fig. 5. Altitude profiles of the burst rate and plasma parameters for season III (orbits $484-560$ ). Similar to Figure 4 . 
tudes, which allows whistler-mode waves to escape from the atmosphere. However, without a measurement of the radial field, we can only speculate that the field actually penetrates to lower altitudes. Moreover, this must occur at some distant location since the bursts in Figure 3 are not continuous through periapsis, and the wave ray path is not locally vertical. (It is important to note that while the wave vector will be vertical for escaping whistler-mode waves due to refraction, the ray path is still mainly field-aligned.) The vertical field must be $>3.6 \mathrm{nT}$ all along a ray path for $100 \mathrm{~Hz}$ whistlermode waves to escape and it is by no means clear that this condition will be satisfied. The field is very weak at lowest altitudes, with a spin-axis component of only a few nT.

Alternatively, the field may be horizontal when the 100 $\mathrm{Hz}$ bursts occur, and a gradient drift instability [Huba, 1992] could be present since the field is perpendicular to the vertical density gradient. In Season III, however, the $100 \mathrm{~Hz}$ bursts are found for much higher densities, where collisions are likely to stabilize the gradient drift instability, and the bursts tend to occur for be vertical fields [Ho et al., 1992], so that the vertical density gradient is field-aligned. While the $100 \mathrm{~Hz}$ bursts detected at higher altitudes during the entry phase could be locally generated, this does not preclude the low altitude bursts detected early in the Pioneer Venus mission and at $\sim 130 \mathrm{~km}$ during entry phase [Strangeway et al., 1993] from being due to atmospheric lightning.

Wideband bursts detected during in entry phase appear to be different than those observed at low altitudes earlier in the mission. First, the bursts tend to occur above $160 \mathrm{~km}$ altitude, and the burst rate is constant with altitude $>160 \mathrm{~km}$. Second, the bursts are a low magnetic field phenomenon. Third, no bursts occur in the $30 \mathrm{kHz}$ channel. During Season III the wideband bursts occurred at low altitudes, in slightly reduced density regions, and also occurred at $30 \mathrm{kHz}$ (see, e.g., Ho et al. [1991]). The ion plasma frequency is $\sim 1.7$ $\mathrm{kHz}$ for an oxygen plasma with density $10^{3} \mathrm{~cm}^{-3}$. Even with Doppler-shift it would be difficult to increase this frequency to $30 \mathrm{kHz}$. We therefore suggest that the wideband waves detected at higher altitudes during the entry phase are ion acoustic mode waves, perhaps generated by solar wind electrons precipitating into the ionosphere. These electrons are also mainly responsible for maintaining the nightside ionosphere during low solar activity [Gringauz et al., 1979; Knudsen et al., 1987]. In this regard, the wideband bursts detected during entry phase cannot be used to infer a lack of association with lightning for the wideband bursts detected earlier in the Pioneer Venus mission, which occurred at lower altitudes and earlier local times.

Acknowledgments. Frederick L. Scarf was the Principal Investigator for the Pioneer Venus Orbiter Electric Field Detector, until his untimely death in 1988. The investigation of plasma waves at Venus would not have been possible without his efforts. We also thank J. Dyer, R. Fimmel, D. Lozier, and M. Smith, as well as the other members of the
Pioneer Venus Project, for their excellent management and operation of the spacecraft. This work was supported by NASA grants NAG2-485 and NAGW-3497.

\section{References}

Gringauz, K. I., M. I. Verigin, T. K. Breus, and T. Gombosi, The interaction of electrons in the optical umbra of Venus with the planetary atmosphere - the origin of the nighttime ionosphere, J. Geophys. Res., 84, 2123-2127, 1979.

Ho, C.-M., R. J. Strangeway, and C. T. Russell, Occurrence characteristics of VLF bursts in the nightside ionosphere of Venus, J. Geophys. Res., 96, 21,361-21,369, 1991.

Ho, C.-M., R. J. Strangeway, and C. T. Russell, Control of VLF burst activity in the nightside ionosphere of Venus by the magnetic field orientation, J. Geophys. Res., 97, 11,673-11,680, 1992.

Huba, J. D., Theory of small-scale density and electric field fluctuations in the nightside Venus ionosphere, $J$. Geophys. Res., 97, 43-50, 1992.

Knudsen, W. C., A. J. Kliore, and R. C. Whitten, Solar cycle changes in the ionization sources of the nightside Venus ionosphere, J. Geophys. Res., 92, 13,391-13,398, 1987.

Russell, C. T., Venus lightning, Space Sci. Rev., 55, 317356, 1991.

Russell, C. T., R. J. Strangeway, J. G. Luhmann, and L. H. Brace, The magnetic state of the lower ionosphere during Pioneer Venus entry phase, Geophys, Res. Lett., this issue, 1993.

Strangeway, R. J., Plasma waves at Venus, Space Sci. Rev., 55, 275-316, 1991.

Strangeway, R. J., An assessment of lightning or in situ instabilities as a source for whistler mode waves in the night ionosphere of Venus, J. Geophys. Res., 97, 12,20312,215, 1992.

Strangeway, R. J., C. T. Russell, and C. M. Ho, Observation of intense wave bursts at very low altitudes within the Venus nightside ionosphere, Geophys, Res. Lett., this issue, 1993.

Theis, R. F., and L. H. Brace, Solar cycle variations of the electron density and temperature in the nightside Venus ionosphere, Geophys, Res. Lett., this issue, 1993.

L. H. Brace, Space Physics Research Laboratory, Department of Atmospheric, Oceanic and Space Science, The University of Michigan, Ann Arbor, MI 48109-2143

C. M. Ho, Jet Propulsion Laboratory, California Institute of Technology, Pasadena, CA 91109-8099

C. T. Russell, and R. J. Strangeway, Institute of Geophysics and Planetary Physics, University of California at Los Angeles, Los Angeles, CA 90024-1567

(Received: July 1, 1993; revised: August 18, 1993; accepted: September 9, 1993) 